\title{
Tandem Femto- and Nanomolar Analysis of Two Protein Biomarkers in Plasma on a Single Mixed Antibody Monolayer Surface using Surface Plasmon Resonance
}

Suhee $\operatorname{Kim}^{\dagger}{ }^{1}$, Jeong Won Park $\dagger^{2}$ Alastair W. Wark ${ }^{*}$, Sung Hwa Jhung ${ }^{1}$, and Hye Jin Lee*1

${ }^{1}$ Department of Chemistry and Green-Nano Materials Research Center, Kyungpook National University, 80 Daehakro, Buk-gu, Daegu-city, 41566, Republic of Korea

${ }^{2}$ Bio-Medical IT Convergence Research Division, SW Contents Research Laboratory, Electronics and Telecommunications Research Institute, 218 Gajeongno, Yuseong-gu, Daejeon, 34129,

Republic of Korea

${ }^{3}$ Centre for Molecular Nanometrology, WestCHEM, Department of Pure and Applied Chemistry, Technology and Innovation Centre, University of Strathclyde, 99 George Street, Glasgow, G1 1RD, $U K$

$\dagger$ S. Kim ${ }^{l}$ and J.W. Park ${ }^{2}$ contributed equally to this work.

*Corresponding authors:

E-mail address: hyejinlee@knu.ac.kr, Tel.+82053950 5336;

E-mail address: alastair.wark@strath.ac.uk,Tel: + 44141548 3084; 


\begin{abstract}
The multiplexed detection of protein biomarkers in plasma present over a range of clinically relevant concentrations continues to be difficult for surface-based bioaffinity detection platforms such as surface plasmon resonance (SPR). As well as nonspecific adsorption, challenges include quantitative comparison between targets whose concentrations differ by orders of magnitude, regenerating SPR chips after plasma exposure as well as the two or four channel limitation of many commercial SPR instruments limiting sample throughput. In this article, we explore an approach where two protein biomarkers alpha-1 antitrypsin (AAT) and Tau 381 are detected in tandem within a single SPR channel at micromolar and femtomolar concentrations respectively. This was achieved by creating a mixed antibody (antiAAT and antiTau) monolayer on the chip surface. Following the adsorption of AAT and/or Tau, further specificity was obtained via the adsorption of a DNA aptamer specific to each target. The detection range for each target was controlled via the relative surface density ratio of each antibody type as well as each aptamer concentration. Calibration measurements were performed in both buffer and spiked plasma with the detection of native concentrations of $~ 39$ $\mathrm{fM}$ (Tau) and $\sim 65 \mu \mathrm{M}$ (AAT) in a human plasma sample. Finally, tandem measurements of both targets within the same SPR signal channel were demonstrated at these very different concentrations.
\end{abstract}

\title{
Introduction
}

The detection of biomarkers in biological fluids (e.g. serum, urine, saliva) has an essential role in disease diagnosis and treatment. However, in many cases such as cancer, autoimmune and neurodegenerative disease the analysis of only one biomarker is insufficient for valid diagnostics, ${ }^{1-5}$ which is not surprising as many proteins operate within complex networks and multi-step pathways. This has led to an increasing interest in developing methodologies that enable comparative analysis of multiple biomarker levels simultaneously. The most established approaches for multiplexed protein biomarker analysis involve either performing a number of 
individual solution-based measurements in parallel (e.g. enzyme linked immunoassays (ELISA's $)^{3,6}$ or developing imaging or scanning methods involving bioreceptors immobilized on an array chip platform. ${ }^{1,7}$ Other techniques for multiplexed immunoanalysis include electrochemical ${ }^{8}$ microring resonators ${ }^{9}$ and a single molecule ELISA digital array assay. ${ }^{10}$

Surface plasmon resonance (SPR) is a well-established technique for the direct analysis of bioaffinity interactions. ${ }^{11}$ However, like all surface-based platforms, the application of SPR to molecular diagnostics in serum and other complex media has continued to be challenging due to non-specific interactions. ${ }^{12}$ Also, while a number of SPR array based measurement configurations are available, ${ }^{13}$ the majority of commercial SPR instruments are typically two and four-channel systems. This can contribute to a significant bottleneck in analysis throughput, especially for plasma diagnostics where recent work by ourselves ${ }^{14-16}$ have found that the SPR chip surface is difficult to regenerate following plasma analysis and cannot be repeatedly used. This issue along with the significant number of repeats and controls required also contributes to an increased number of SPR chips typically required for plasma analysis. It would also potentially help measurement throughput if tandem measurements could be performed within a single channel on platforms where the opportunity to create multiple spatially separated probe regions is limited. This concept has been little explored in the literature with examples of controlling the surface density of mixed DNA probes ${ }^{17}$ but we are not aware of a study demonstrating the application of mixed monolayers of two antibody probes.

In this paper, we explore the creation of mixed bioreceptor monolayers on a single sensing surface to address the challenge of increasing sample analysis throughput. Specifically, we demonstrate that it is possible to achieve wide dynamic range sensing of two different protein 
biomarkers at clinically relevant concentrations within the same SPR detection channel. This was achieved by creating a dual antibody probe layer as part of a multiplexed sandwich assay with DNA aptamers introduced to the SPR chip surface following exposure to the target proteins. Used in this study was (i) Tau protein (the Tau-381 isoform), which is important in neurodegenerative diseases such as Alzheimer's, ${ }^{18}$ and (ii) alpha-1 antitrypsin (AAT), where high or deficient ${ }^{19}$ levels are associated with various conditions such as lung ${ }^{20}$ and Alzheimer's disease. $^{21}$ The selection of Tau and AAT as a model system was also based on each having very different native concentrations (femtomolar ${ }^{22}$ and micromolar ${ }^{23}$ respectively) in the plasma of healthy patients as well as each having established high affinity antibody/aptamer assay pairings. ${ }^{15,16}$ In addition to creating a mixed monolayer of antiTau and antiAAT on the SPR chip surface, the concentration of the aptamers specific to each target were also optimized in order to detect both protein targets on the same chip surface at very different concentrations. A series of spiked plasma measurements were performed with the resulting calibration utilized to determine the native concentrations of both target proteins present in a human plasma sample.

\section{Experimental Section}

Further experimental details are provided in the Supporting Information. Briefly, recombinant Human Tau381 full length protein (Tau) and tau antibody (antiTau) were acquired from Abcam. Also, both alpha-1 antitrypsin (AAT) and alpha-1 antitrypsin antibody (antiAAT) were obtained from R\&D systems. A $5^{\prime}$-amine modified DNA aptamer ${ }^{24}$ specific to Tau, whose sequence is 5'-H ${ }_{2} \mathrm{~N}-G C G G A G C G T G G C A G G-3 '$ and a 5'-amine modified DNA aptamer ${ }^{25}$ specific to AAT, whose sequence is 5'-H H $_{2}$-GGG GCA CGT ACG GGC ATC ATA ACA ACA GGC GTG CCC C-3' were purchased from Integrated DNA Technologies. Also, 1-ethyl-3-(3dimethylaminopropyl)carbodiimde hydrochloride $\quad$ (EDC, $\quad$ Thermo), N- 
hydroxysulfosuccinimide (NHSS, Thermo Scientific), 11-mercaptoundecanoic acid (MUA, Sigma-Aldrich), and plasma from human (Sigma-Aldrich) were all used as received. The proteins used for control measurements were immunoglobulin G (IgG; Sigma-Aldrich) and bovine serum albumin (BSA; Calbiochem). All in-situ SPR bioaffinity measurements were performed using a Biacore 3000 with a flow rate of $5 \mu \mathrm{L} / \mathrm{min}$ employed for all experiments. Different concentrations of AAT or Tau solution were individually flowed over the functionalized SPR chips for a minimum of $1 \mathrm{~h}$ to ascertain a steady-state surface coverage for both specific and non-specific binding experiments. Plasma samples were also injected across the antibody chip surface for at least $1 \mathrm{~h}$ followed by rinsing with PBS buffer. The DNA aptamers for either AAT or Tau were suspended at various concentrations in 1x PBS buffer $(\mathrm{pH}$ 7.4) and flowed over the SPR chip for 40 mins followed by washing with PBS buffer for 15 mins. For AAT or Tau measurements in undiluted or 1,000 times diluted plasma the antibody surface could not be completely regenerated after a single measurement. Thus, all plasma measurements reported were performed with a freshly prepared chip surface. Each data point reported is the average of a minimum of three repeat measurements.

\section{Results and Discussion}

Detection of AAT and Tau Proteins using a Mixed Antibody Chip: The strategy for detecting two biomarkers (Tau and AAT) simultaneously in a single detection microchannel is outlined in Figure 1. The gold SPR chip surface was first functionalized with a monolayer of 11mercaptoundecanoic acid (MUA) to which the corresponding antibodies (antiTau and antiAAT) were covalently coupled via EDC/NHSS linking chemistry via an amine group on the antibody surface with the relative surface coverage of each antibody systematically varied. Specific adsorption measurements for both targets were performed first in buffer and then demonstrated 
in spiked human plasma solution. The SPR detection signals for both targets were then further enhanced by flowing the corresponding DNA aptamer specific to either Tau or AAT (in buffer solution) across the chip surface. This binds to a different epitope site on the respective target to complete the formation of a sandwich assay.

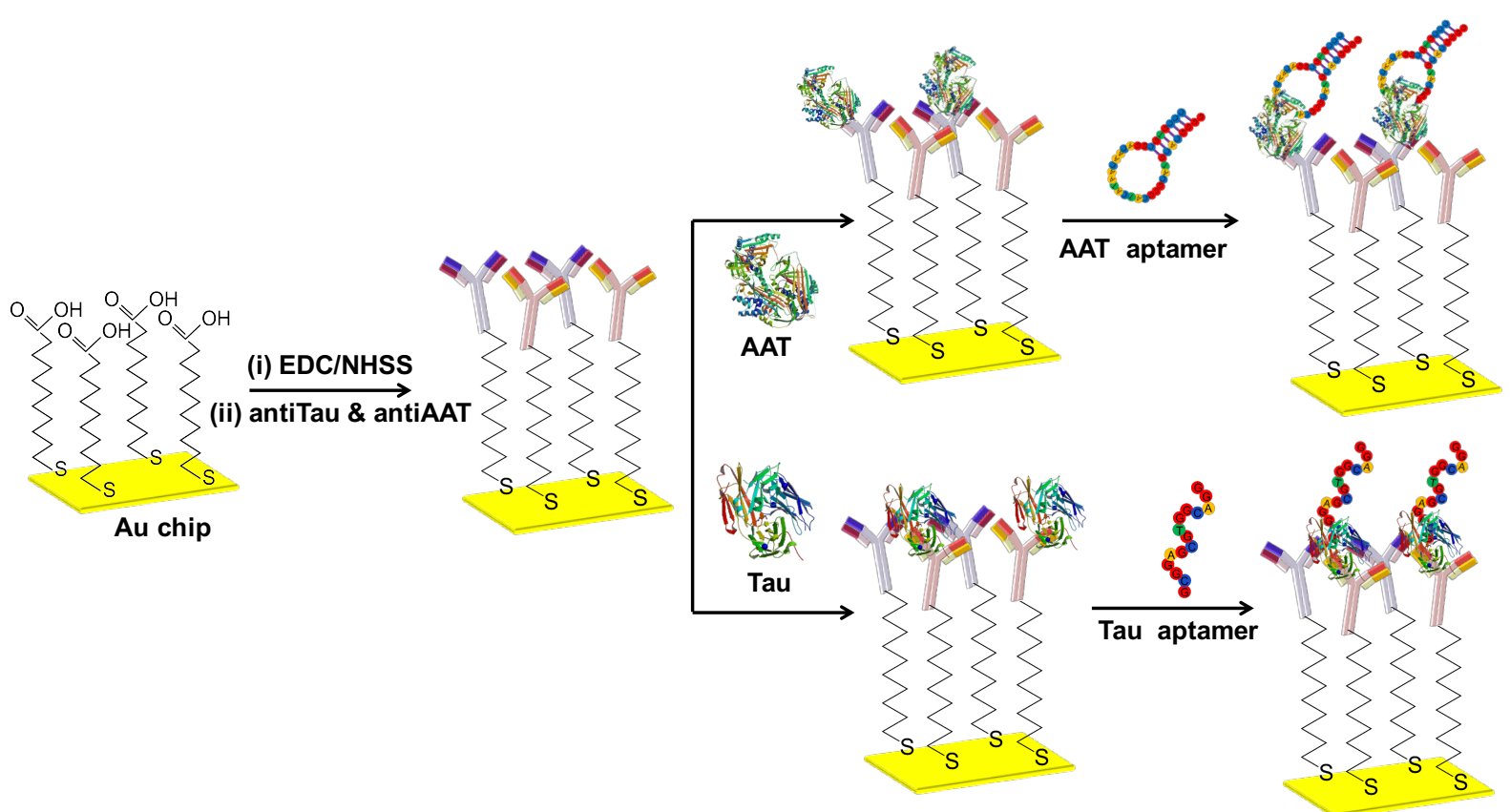

Figure 1. Schematic overview of strategy for dual detection of AAT and Tau proteins on a single surface. Mixed monolayers of antiAAT and antiTau were first immobilized on a MUA modified SPR chip. Specific adsorption of AAT and Tau proteins were monitored followed by the subsequent binding of AAT aptamer and Tau aptamer. Images representative of AAT and Tau proteins are reproduced from refs ${ }^{26}$ and ${ }^{27}$, respectively.

In order to successfully detect both targets quantitatively that differ in both concentration and the strength of the affinity interaction between the target and respective probes, it is essential that the relative surface densities of both antibodies in the mixed monolayer are well-controlled. This was achieved during the covalent coupling of the capture antibodies onto the SPR chip where the total antibody concentration introduced to the microfluidic chip was fixed at $10 \mu \mathrm{M}$ and the stoichiometric ratio of both antiAAT and antiTau were systematically varied between 0 and $100 \%$. This approach does assume that the resulting surface density of each antibody on the SPR chip closely follows the bulk ratios utilized in the reaction solution. 
The sensing performance of SPR chips incubated with different molecular ratios of antiAAT and antiTau were investigated by performing an extensive series of real-time SPR measurements systematically varying both the AAT and tau target concentration and the mixed antibody monolayer composition. Some representative SPR response curves for these measurements are shown in the supporting information (Figure S1). Also, a series of $\Delta \mathrm{R}$. U. plots were generated (see Figure S2) by systematically exposing a range of AAT or Tau concentrations to nine different mixed monolayer chip surfaces. These were systematically varied from $100 \%$ coverage of only one antibody and diluted down to mixtures containing $20 \%$ of the antibody specific to the target species being monitored.

Figure 2 represents a succinct approach to comparing the results obtained for the various combinations of mixed antibody monolayers and protein target concentrations distributed across the eighteen data series shown in Figure S2. Each SPR chip monolayer configuration was analyzed to establish a target concentration at which the R.U. response is close to saturation (i.e. beyond the linear response with minimal further change in SPR signal with increasing concentration) is extracted for each mixed antibody monolayer combination. This concentration is then normalized with respect to the concentration associated with the signal saturation for the $100 \%$ antiAAT or antiTau monolayer creating the y-axis ratio in Figure 2 ranging between 0 and 1 . The resulting plot highlights how the availability of surface binding sites and sensing performance vary as a function of the mixed monolayer composition. For example, at an antiAAT : antiTau ratio of $20: 80$, the AAT detection saturation concentration is about $40 \mathrm{nM}$ compared to $\sim 120 \mathrm{nM}$ for Tau detection. These are normalized respectively to saturation concentrations of $500 \mathrm{nM}$ and $200 \mathrm{nM}$ for $100 \%$ antiAAT and $100 \%$ antiTau surfaces. 


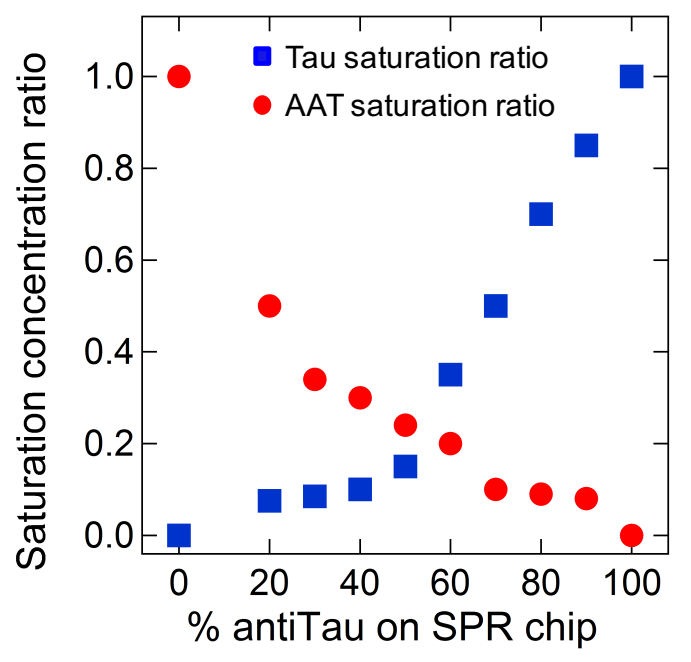

Figure 2. Plot of the saturation concentration ratio for AAT and Tau binding onto a range of mixed monolayer surfaces.

As the relative surface densities of both capture antibodies vary, Figure 2 confirms that each target protein can still be selectively measured on a multiplexed chip surface and highlights how the measurement dynamic range becomes more limited as the density of the antibody capture agent is reduced. It is clear that a more marked difference between $100 \%$ and $80 \%$ antiAAT coverage is observed compared to the same change in antiTau coverage with the Tau detection dynamic range decreasing more gradually as the fractional coverage of antiTau is reduced. The differences in the two plots in Figure 2 may be associated with factors such as the antibody surface coupling efficiency affecting the relative surface coverage of each antibody or differences in binding affinity. The adsorption coefficient $\left(K_{\text {ads }}\right)$ for AAT onto a $100 \%$ antiAAT layer was determined to be $7.95( \pm 0.4) \times 10^{6} \mathrm{M}^{-1}$ (Figure S3a) while a $K_{\text {ads }}$ of $3.9( \pm 0.3) \times 10^{7} \mathrm{M}^{-1}$ was determined for Tau adsorption onto a $100 \%$ antiTau surface (see Figure $\mathrm{S} 3 \mathrm{~b})$. These values agree well with previous measurements performed by us ${ }^{15,16}$ and others. ${ }^{28}$

Sandwich assay on mixed monolayer chips. The study on the different mixed monolayer configurations clearly showed that both Tau and AAT could be detected at nanomolar 
concentrations on surfaces featuring a mixture of antibodies specific to both targets. In order to further improve sensitivity and selectivity, a sandwich assay was applied and we investigated this on three different surfaces with antiAAT:antiTau ratios of 30:70, 50:50, and 70:30. Figure 3 compares a series of plots where a range of concentrations of DNA aptamer specific to either AAT (3a-c) or Tau (3d-f) was flowed across different SPR chips that had been previously exposed to a fixed protein target concentration. The choice of fixed AAT or Tau concentration was based on the results used to generate Figure 2, selecting a value that corresponds to a high fractional surface coverage. Each $\Delta$ R. U. data point in Figure 3 was obtained by taking the average change in R. U. signal comparing before and after target binding in buffer solution only (see Figure S4 for corresponding R. U. curves). In each chip, target binding is monitored in two of the available four measurement channels available on each SPR chip with the remaining two SPR channels used to perform a series of control measurements. The final $\Delta \mathrm{R}$. U. values plotted is the difference between the averaged detection and control channel signals acquired over three repeat sets of measurements. A summary of the different negative control (NC) measurements used in shown in the supporting information (Table S2) involving repeat measurements in the absence of either Tau or AAT target (NC1) or instead introducing BSA (NC2) or IgG (NC3) in place of the target protein. Representative R. U. plots from NC control channels are also shown in the supporting information (Figure S5). In the measurements performed, we also saw no non-specific interaction between the Tau aptamer and antiAAT and AAT with the same applied in reverse with AAT aptamer not interacting with antiTau or Tau. 

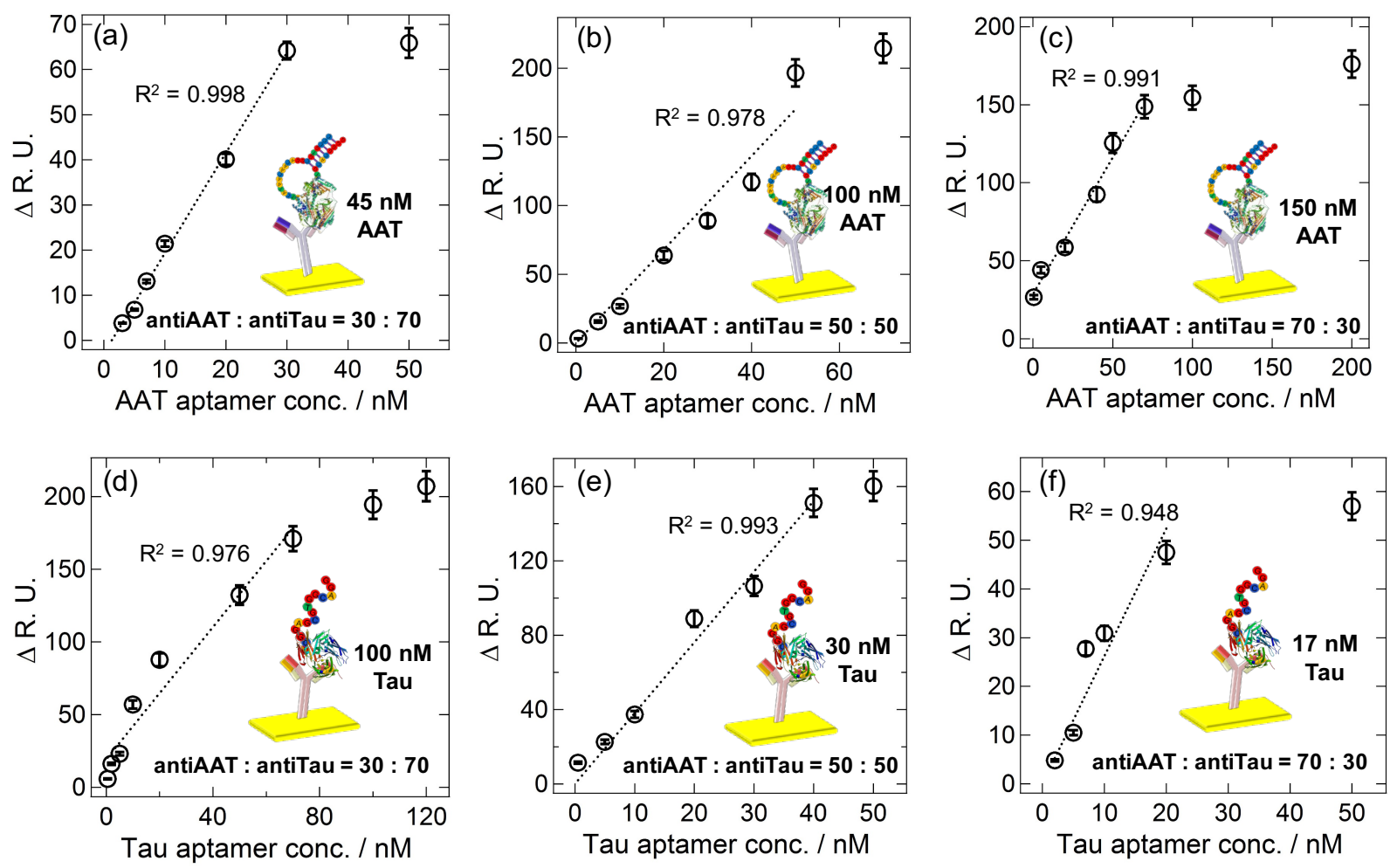

Figure 3. Comparing change in SPR signals obtained upon introducing different concentrations of DNA aptamer specific to AAT (a, b, c) and Tau (d, e, f) on chip surfaces prepared with three different antiAAT : antiTau ratios of 30:70, 50:50 and 70:30. In each case, the chip surface was first exposed to a fixed target protein concentration (which is stated in each plot) for a minimum of $1 \mathrm{hr}$ prior to introducing the aptamer. The dotted line in each plot indicates the linear SPR response range region.

The results in Figure 3 provides evidence that both the DNA aptamer and antibody pairings for the AAT and Tau protein targets bind simultaneously via different epitope sites. Similar measurements were also performed on $100 \%$ antiAAT and $100 \%$ antiTau surfaces (see Figure S6). These represent the largest fractional antibody coverages possible in both cases and it can be seen that a linear SPR response is obtained for an aptamer concentration range between $\sim 10-200 \mathrm{nM}$, above which the signal does not change significantly. For the mixed monolayer measurements in Figure 3 it can be seen that the $\Delta \mathrm{R}$. U. response is saturated at considerably lower DNA aptamer concentrations e.g. for the 70:30 antiAAT : antiTau surface, AAT and Tau aptamer concentrations of $\sim 100 \mathrm{nM}$ and $\sim 20 \mathrm{nM}$ would be sufficient to produce close to the maximum enhancement of the SPR signal upon sandwich assay formation. In addition, as the 
antibody ratio is changed to 50:50 and 30:70 the aptamer concentrations associated with signal saturation are lower for AAT and higher for Tau detection.

Further experiments were then performed to tailor the measurement range for both target proteins by controlling both the surface antibody ratio and DNA aptamer concentrations. A significant challenge is being able to detect both targets on the same surface at very different concentration ranges. In clinical samples, AAT samples are typically at $\sim 24-35 \mu \mathrm{M}$ levels, ${ }^{23,29}$ while for Tau measurements in healthy patients, analysis capabilities in the $100 \mathrm{fM}$ range is required. $^{22}$ To achieve dual detection on the same surface at such large differences in concentration, an antiAAT : antiTau ratio of $30: 70$ was selected. Figure S7 shows the results of measurements performed at a fixed concentration of (a) $30 \mathrm{nM}$ AAT and (b) $100 \mathrm{fM}$ Tau with the target specific DNA aptamer concentration systematically varied in each case. Based on these results, AAT aptamer and Tau aptamer concentrations of $30 \mathrm{nM}$ and $100 \mathrm{nM}$ respectively were established for performing clinically relevant measurements in human plasma.

Plasma measurements. Prior to performing the multiplexed detection of both target species, a series of individual measurements were performed where Tau or AAT were spiked into human plasma over a range of concentrations. These results are shown in Figure 4 using the optimum antiAAT : antiTau surface monolayer ratio of $30: 70$ and concentrations of the specific DNA aptamers established earlier. Representative R. U. plots are shown in figs. 4(a) and 4(c) for both spiked, nonspiked and control measurements in plasma. In the corresponding $\Delta \mathrm{R}$. U. plots in Figure 4(b) and 4(d) a set of measurements acquired in buffer only is shown alongside the plasma data series. In both sets of data the $\Delta$ R. U. signals were consistently higher for plasma, 
which can be associated with non-specific adsorption. In the case of the Tau measurements (Figure 4d) both the plasma and buffer series indicate similar linearity indicated by the dotted lines (1.14 versus $0.96 \Delta$ R. U./fM for plasma and buffer respectively). This suggests the amount of nonspecific adsorption in the nondiluted plasma is relatively constant for all measurements. For the diluted plasma measurements in Figure 4(b), there is a bigger difference between the plasma and buffer slope fits (1.04 versus $1.64 \Delta \mathrm{R}$. U./nM respectively). Thus, in both data sets the linear fit of the spiked plasma measurements was used to estimate the native AAT and Tau concentrations. This is described in more detail in the supporting information with values of $39( \pm 5) \mathrm{fM}$ for Tau and $65( \pm 8) \mu \mathrm{M}$ for AAT obtained. Both of these values are in the range expected, as discussed above, with the AAT concentration also taking into account the 1000-fold dilution of the plasma stock prior to measurement and spiking. 

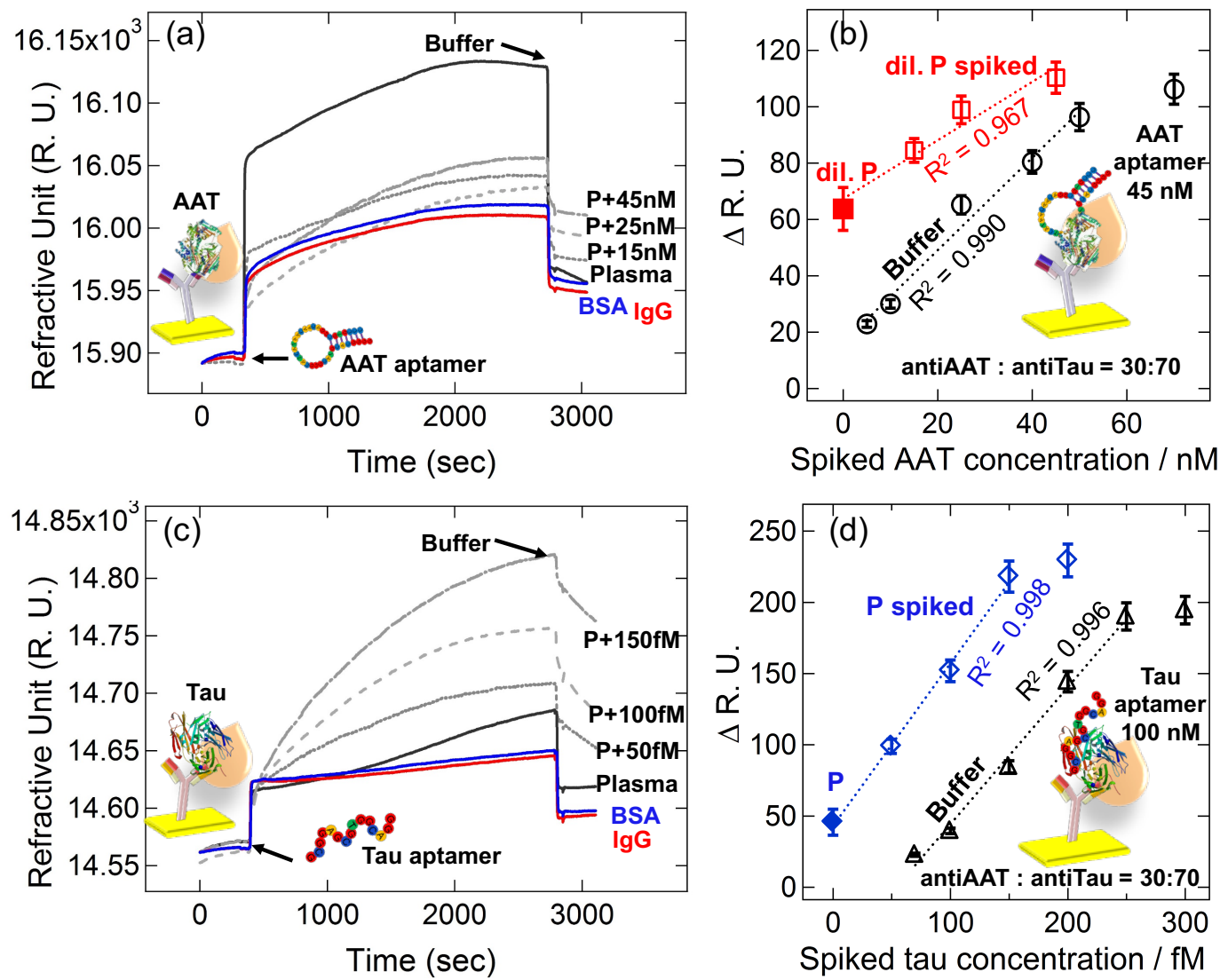

Figure 4. SPR measurements in plasma for (a), (b) AAT and (c), (d) Tau measurements in both spiked and native plasma (P) performed on a antiAAT : antiTau 30 : 70 chip surface. Representative SPR sensorgrams are shown in (a) and (c) following exposure to plasma (both spiked and native) alongside examples of negative controls BSA, IgG spiked at $25 \mathrm{nM}$ in (a) and $100 \mathrm{fM}$ in (c). For the AAT samples, the plasma was first diluted 1000-fold in buffer before spiking, while for the Tau measurements undiluted plasma is used directly. In (b) and (d), the dotted line is a guide for the linear response range with the plasma only $(\mathrm{P})$ and spiked $\mathrm{P}$ data points indicated separately.

A final set of experiments were performed to demonstrate the dual detection of both targets concurrently within the same SPR detection channel. This was achieved by exposing the 30:70 antiAAT:antiTau surface to a mixture of both targets for at least one hour before then sequentially flowing each of the target aptamers across the SPR chip. Figure 5(a) shows representative R. U. plots in 1000-fold diluted plasma for both plasma only as well as spiked at two different AAT and Tau concentrations. Additional R. U. sensorgrams are also shown in 
Figure S9 (supporting information) for the measurements in spiked buffer and nondiluted plasma.

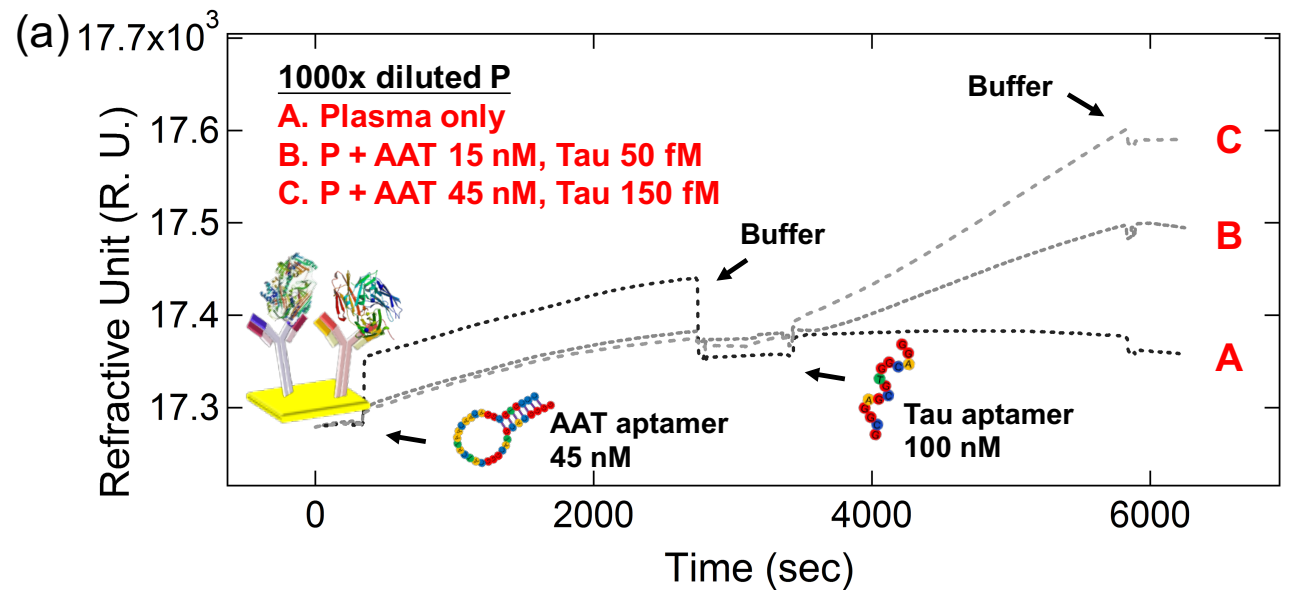

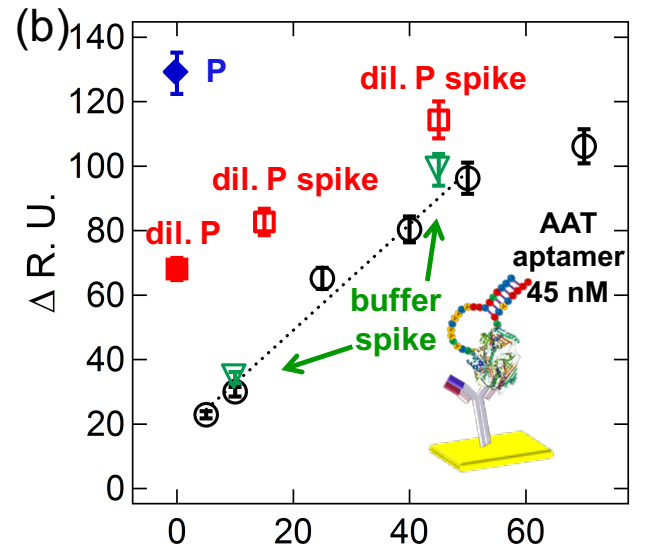

Spiked AAT concentration / nM

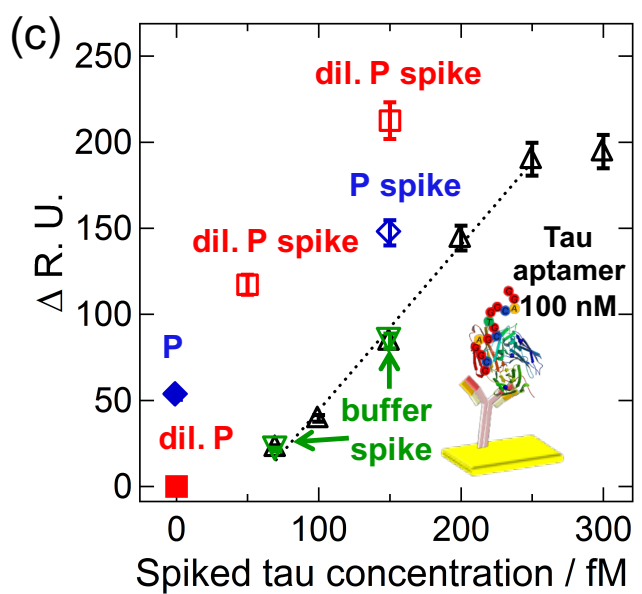

Figure 5. Comparison of concurrent SPR measurements of AAT and Tau performed in plasma (P), 1000x-diluted plasma (dil. P) and buffer. (a) R. U. plots performed on a antiAAT : antiTau 30 : 70 chip surface which was exposed to 1000-fold diluted plasma, both spiked and plasma only, for at least one hour prior to the sequential introduction of aptamers specific to AAT and Tau. (b) $\Delta R$. U values obtained for AAT and (c) Tau obtained for dual target measurements. The data series acquired in buffer in Figure 4(b) and 4(d) are shown again here (black data points) for comparison with the dual detection data points in plasma (blue), dil. P (red) and buffer (green).

The $\Delta$ R. U. values obtained from analysis of the sensorgrams (in Figure 5(a) and Figure S9) are shown in Figure 5(b) and (c), which focus on AAT and Tau detection separately. Also shown alongside the dual detection measurements are the buffer calibration values from Figure 
4(b) and (d). The dual spiked buffer experiments were performed at concentrations of (10 nM AAT, $70 \mathrm{fM} \mathrm{Tau}$ ) and (45 nM AAT, $150 \mathrm{fM}$ Tau) and the $\Delta$ R. U. values obtained show good overlap with the previous single target measurements. This supports the assertion that there is no significant desorption of the Tau target during the AAT aptamer binding step before introducing the Tau aptamer. In the subsequent analysis of the diluted plasma (15 nM AAT, 50 $\mathrm{fM} \mathrm{Tau}$ ) and (45 nM AAT, $150 \mathrm{fM} \mathrm{Tau}$ ) the AAT $\Delta \mathrm{R}$. U. values are similar to that obtained in Figure 4(b), while the signal using nondiluted plasma is saturated beyond the SPR linear response range. For Tau detection, a $\Delta \mathrm{R}$. $\mathrm{U}$. of close to zero is obtained for the diluted plasma (without spiking), which was expected as the native concentration here will be too low to detect. Good reproducibility compared to that observed in Figure 4(d) was also observed. These results confirm that it is possible to perform more complex tandem measurements involving at least two different target species within a single SPR detection channel.

\section{Conclusions}

In this article, we demonstrate that two protein biomarker targets can be detected together on the same SPR chip surface with both species present at target concentrations differing by several orders of magnitude. This was achieved by controlling and optimizing both the ratio of the capture antibodies on the chip surface along with the concentration of the secondary DNA aptamer capture agents used. As a result, it was possible to detect AAT at nanomolar concentrations alongside Tau 381 at femtomolar concentrations within the same SPR detection channel. A motivation for this work is that following a measurement in plasma the SPR chip surface is difficult to regenerate. For successful measurements in plasma it is essential that control measurements are also acquired in parallel reference channels alongside the target. This fact, combined with many high-end SPR devices limited to four detection channels, 
significantly reduces multiplexing capabilities as well as adding cost and time in the number of SPR chips required. The methodology developed here helps to address this problem. In future, this work will be extended to more complex analyses such as the introduction of plasma at a particular dilution followed by a specific antibody or aptamer and repeating this cycle from highly diluted to nondiluted plasma samples. For example, this could be necessary to help quantify differences between two targets occurring between healthy and diseased patient samples as performing such measurements across the femto- to micro-molar range is still a significant challenge.

\section{Acknowledgement}

This research was supported by the National Research Foundation (NRF) of Korea funded by the Ministry of Science, ICT, and Future Planning (grant number: NRF-2016R1A2B4012026).

\section{Supporting Information}

Further experimental details include materials used, fabrication of mixed antibody biochips as well as SPR measurements. Figures S1-S9, Table S1 is also included. This material is available free of charge via the Internet at http://pubs.acs.org.

\section{References}

(1) Kingsmore Stephen, F. Nat. Rev. Drug Discov. 2006, 5, 310-320.

(2) Trojanowski, J. Q.; Vandeerstichele, H.; Korecka, M.; Clark, C. M.; Aisen, P. S.; Petersen, R. C.; Blennow, K.; Soares, H.; Simon, A.; Lewczuk, P.; Dean, R.; Siemers, E.; Potter, W. Z.; Weiner, M. W.; Jack, C. R., Jr.; Jagust, W.; Toga, A. W.; Lee, V. M. Y.; Shaw, L. M. Alzheimers Dement. 2010, 6, 230-238.

(3) Tighe, P. J.; Ryder, R. R.; Todd, I.; Fairclough, L. C. Proteomics Clin. Appl. 2015, 9, 406422. 
(4) Diamandis, E. P. J. Natl. Cancer Inst. 2010, 102.

(5) Fu, Q.; Zhu, J.; Van Eyk, J. E. Clin. Chem. 2010, 56, 314.

(6) Reynolds, M. A.; Kirchick, H. J.; Dahlen, J. R.; Anderberg, J. M.; McPherson, P. H.; Nakamura, K. K.; Laskowitz, D. T.; Valkirs, G. E.; Buechler, K. F. Clin. Chem. 2003, 49, 17331739.

(7) Lee, H. J.; Wark, A. W.; Corn, R. M. Analyst 2008, 133, 975-983.

(8) Wei, F.; Patel, P.; Liao, W.; Chaudhry, K.; Zhang, L.; Arellano-Garcia, M.; Hu, S.; Elashoff, D.; Zhou, H.; Shukla, S.; Shah, F.; Ho, C.-M.; Wong, D. T. Clin. Cancer Res. 2009, 15, 4446.

(9) Washburn, A. L.; Luchansky, M. S.; Bowman, A. L.; Bailey, R. C. Anal. Chem. 2010, 82, 69-72.

(10) Rissin, D. M.; Kan, C. W.; Campbell, T. G.; Howes, S. C.; Fournier, D. R.; Song, L.; Piech, T.; Patel, P. P.; Chang, L.; Rivnak, A. J.; Ferrell, E. P.; Randall, J. D.; Provuncher, G. K.; Walt, D. R.; Duffy, D. C. Nat. Biotechnol. 2010, 28.

(11) Homola, J. Chem. Rev. 2008, 108, 462-493.

(12) Masson, J.-F. ACS Sensors 2017, 2, 16-30.

(13) Linman, M. J.; Abbas, A.; Cheng, Q. Analyst 2010, 135, 2759-2767.

(14) Jang, H. R.; Wark, A. W.; Baek, S. H.; Chung, B. H.; Lee, H. J. Anal. Chem. 2014, 86, 814-819.

(15) Kim, S.; Lee, H. J. Anal. Chem. 2015, 87, 7235-7240.

(16) Kim, S.; Wark, A. W.; Lee, H. J. Anal. Chem. 2016, 88, 7793-7799.

(17) Nakano, S.-i.; Kanzaki, T.; Nakano, M.; Miyoshi, D.; Sugimoto, N. Anal. Chem. 2011, 83, 6368-6372.

(18) Liao, P.; Yu, L.; Kuo, C.; Lin, C.; Kuo, Y. Proteomics Clin. Appl. 2007, 1, 56.

(19) Rachelefsky, G.; Hogarth, D. K. J. Allergy Clin. Immunol. 2008, 121, 833.

(20) Linja-aho, A.; Mazur, W.; Toljamo, T.; Nieminen, P.; Ohlmeier, S.; Rönty, M.; Kinnula, V. L. APMIS 2013, 121, 11-21.

(21) Lista, S.; Faltraco, F.; Prvulovic, D.; Hampel, H. Prog. Neurobiol. 2013, 101-102, 1-17.

(22) Zetterberg, H.; Wilson, D.; Andreasson, U.; Minthon, L.; Blennow, K.; Randall, J.; Hansson, O. Alzheimers Res. Ther. 2013, 5, 1-3.

(23) Ferrarotti, I.; Scabini, R.; Campo, I.; Ottaviani, S.; Zorzetto, M.; Gorrini, M.; Luisetti, M. Transl. Res. 2007, 150, 267-274.

(24) Krylova, S. M.; Musheev, M.; Nutiu, R.; Li, Y.; Lee, G.; Krylov, S. N. FEBS Lett. 2005, 
$579,1371-1375$.

(25) Greving, M.; Woodbury, N. Non-random aptamer libraries and methods for making 2007, WO2007109067 (A2).

(26) Huntington, J. A.; Yamasaki, M.; RCSB PDB (www.rcsb.org): PDB ID 3T1P, 2011.

(27) Cehlar, O.; Skrabana, R.; Novak, M.; RCSB PDB (www.rcsb.org): PDB ID 4TQE, 2015.

(28) Piotrowska, U.; Adler, G. Scand. J. Immunol. 2005, 62, 521-527.

(29) Sun, Y. X.; Minthon, L.; Wallmark, A.; Warkentin, S.; Blennow, K.; Janciauskiene, S. Dement. Geriatr. Cogn. Disord. 2003, 16, 136-144. 
For TOC only

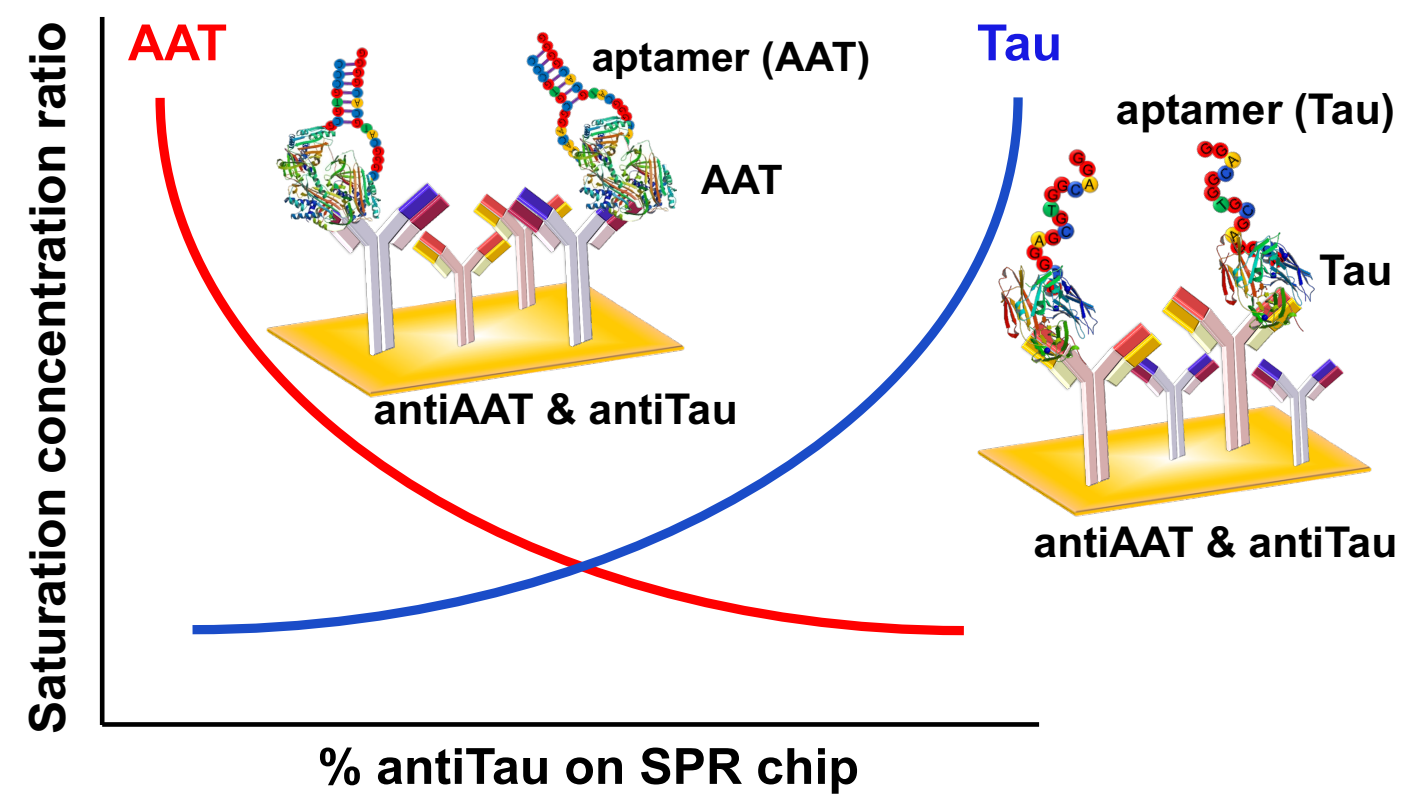

